\title{
Effect of thermal cycling on performance of Poly(3-hexylthiophene) Transistors
}

Brian A. Mattis, Paul C. Chang, and Vivek Subramanian

Department of Electrical Engineering and Computer Sciences, University of California, Berkeley Berkeley, CA 94720, U.S.A.

\section{ABSTRACT}

We present the results of studies on the electrical and physical modifications to Poly(3hexylthiophene), upon thermal annealing. Thermally-induced performance modifications and thermal stability of polythiophene thin film transistors are explored. We observe substantial mobility improvements in devices annealed at low temperatures $\left(<80^{\circ} \mathrm{C}\right)$, as well as increases in on/off ratios by two orders of magnitude at moderate anneal temperatures $\left(\sim 120^{\circ} \mathrm{C}\right)$. We document changes in conductivity, mobility, on current, and on/off ratio with anneal temperature and total thermal budget. In conjunction with material analysis, we develop qualitative models for the mechanisms involved in the annealing/ degradation processes. Hence, this study provides a comprehensive analysis of the effect of thermal cycling of polythiophene TFTs on various device performance metrics, and identifies the relevant thermal limits and failure mechanisms.

\section{INTRODUCTION}

In recent years, there has been great interest in organic semiconductor devices, driven by their potential use in low-cost flexible displays and disposable electronics applications. In particular, there has been interest in soluble organic semiconductors, since these may potentially be used to form low-cost all-printed circuits by eliminating the need for many of the major semiconductor-manufacturing cost points, including lithography, PVD and CVD, plasma etching, and the waste management costs associated with subtractive processing. Of the various soluble organic semiconductors currently in use, poly(3-hexylthiophene) has been the most extensively studied, due to its easy synthesis and relatively high mobility $\left(\sim 10^{-2} \mathrm{~cm}^{2} / \mathrm{V}-\mathrm{s}\right)[1]$.

While there have been several demonstrations of polythiophene-based TFTs, there have been few studies on the thermal stability the same. This is an important topic for various reasons. First, polythiophene-based devices will be likely exposed to elevated temperatures during the fabrication process, due to the annealing requirements of other layers. Second, thermal cycling studies provide crucial insights into device lifetime and stability. Finally, given the sensitivity of polythiophene to oxygen and moisture absorbed during the fabrication process, it is expected that thermal treatments may be used to recover some of the lost performance through thermally-induced expulsion of absorbed contaminants. Here, we explore the effects of thermal cycling on performance of polythiophene devices, and relate the measured performance variation to associated physical phenomena, including oxygen and moisture de-doping, as well as irreversible thermally-induced deterioration of the semiconductor material.

\section{EXPERIMENTAL DETAILS}

All experiments were performed on substrate-gated polythiophene TFTs with thermal $\mathrm{SiO}_{2}$ dielectrics. This ensured good repeatability and elimination of experimental artifacts originating from other, less well-behaved, structures. Au contacts were evaporated onto a 100nm thermal $\mathrm{SiO}_{2}$ layer on an n-doped $\mathrm{Si}$ wafer. Poly(3-hexylthiophene) (P3HT) with greater than 98.5 
regioregularity was obtained from Aldrich and mixed at $0.8 \mathrm{wt} \%$ in chloroform. The solution was spin-cast in a nitrogen environment at 6000RPM to achieve a film thickness of approximately $100 \mathrm{~nm}$. Six inert-ambient curing temperatures were explored over the temperature range from $40^{\circ} \mathrm{C}$ to $140^{\circ} \mathrm{C}$. For each of these temperatures, four different anneal times between 1min and 1000mins were explored. All experiments were performed on multiple samples, each containing multiple pre-measured and mapped devices, to minimize statistical variations, and to enable pre- vs. post- exposure analysis. The data set therefore consisted of measurements from more than 200 samples.

Electrical measurements were performed in a nitrogen environment. Gate and drain voltages were swept between $5 \mathrm{~V}$ and $-35 \mathrm{~V}$, and both $\mathrm{I}_{\mathrm{D}}-\mathrm{V}_{\mathrm{G}}$, and $\mathrm{I}_{\mathrm{D}}-\mathrm{V}_{\mathrm{D}}$ measurements were made. For all data presented, the post annealing data was normalized to the pre-annealing data to enable proper comparisons. To correlate the measured electrical variations in device performance to physical effects, various materials analysis tests were performed, including RBS (for background oxygen level measurement) and AFM (for surface roughness measurement). Base mobilities averaged $\sim 10^{-3} \mathrm{~cm}^{2} / \mathrm{V}$-s and on/off ratios were $\sim 10^{3}$.

\section{RESULTS AND DISCUSSION}

Variations in device electrical performance as a function of anneal temperature and time are shown in Figures 1 and 2. As is evident from the various regions of increasing and decreasing performance, multiple effects occur, with different effects dominating in the various temperature regimes. Figure 1 indicates that saturation mobility can be more than doubled via long duration low-temperature anneals $\left(60^{\circ} \mathrm{C}\right)$. Additionally, on/off ratios for $\mathrm{P} 3 \mathrm{HT}$ can be increased by almost 3 orders of magnitude by using long duration anneals at $120^{\circ} \mathrm{C}$ (fig 2). Above this temperature damage to the film occurs, resulting in a decrease in both on/off ratio and mobility. To account for the effect of threshold voltage shifts, on-off ratio was calculated by shifting the I$\mathrm{V}$ characteristics to match the $\mathrm{V}_{\mathrm{T}}$ 's, and the saturation mobility was calculated using:

$$
\mu_{\mathrm{SAT}}=\mathrm{g}_{\mathrm{m}}^{2} / 2 \mathrm{I}_{\mathrm{D}} \mathrm{C}_{\mathrm{OX}}(\mathrm{W} / \mathrm{L})
$$

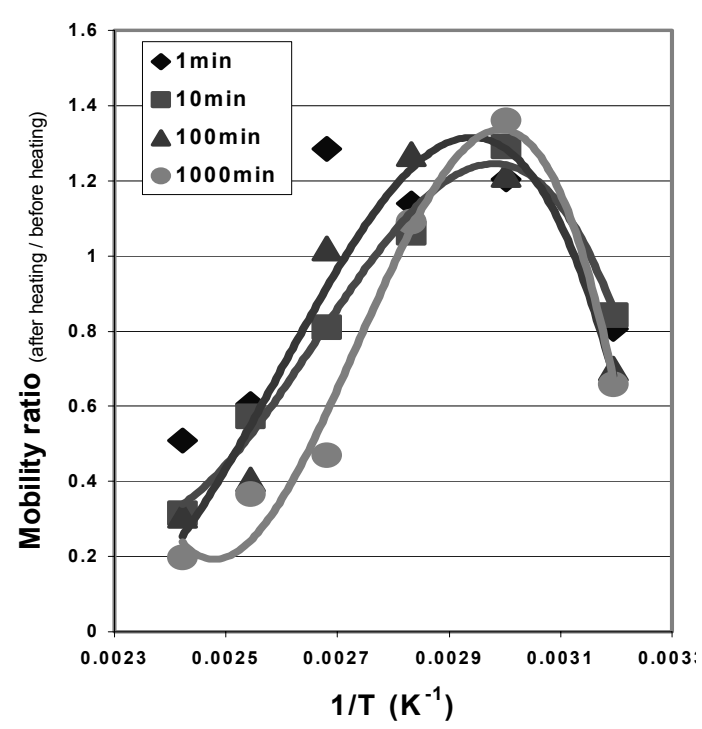

Figure 1: Variation in saturation mobility with thermal cycling

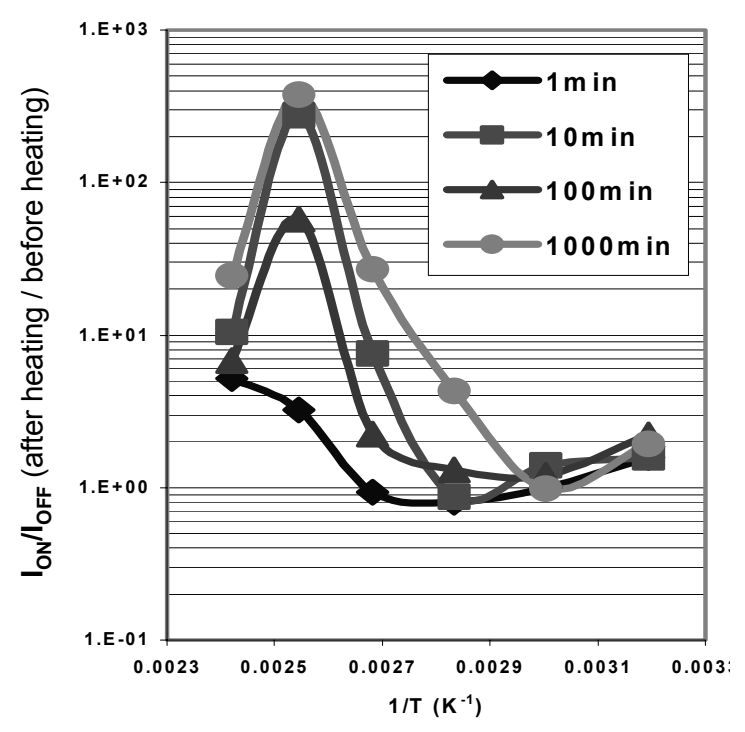

Figure 2: Variation in on-off ratio with thermal cycling 
From the above figures, it is apparent that there are three regions of operation:

- Up to $\sim 60^{\circ} \mathrm{C}$, the mobility increases, while the on-off ratio is mainly unchanged.

- At moderate temperatures, $60^{\circ} \mathrm{C}-120^{\circ} \mathrm{C}$, there is a dramatic increase in on-off ratio, while mobility is degraded continually with increasing temperature.

- At high temperatures, $>120^{\circ} \mathrm{C}$, both mobility and on-off ratio are degraded.

To explain the variations of device performance in the three regimes, we introduce three different phenomena, and correlate the electrical results to materials analyses. The three concepts used to explain the phenomena seen are the oxygen dedoping of the P3HT film, the torsion of the polymer chain near melting temperatures, and the elimination of moisture / solvent from the film due to thermal evolution.

\section{Oxygen Dedoping}

During the production and solution preparation of the P3HT material, the solution absorbs oxygen from the environment. Therefore, despite inert ambient-based device fabrication and electrical characterization, significant concentrations of oxygen are expected to be embedded in the films. This oxygen dopant serves to increase the conductivity of the films through the production of localized states within the $\pi-\pi^{*}$ gap $[2,3]$. This increases the off-current of the transistors, reducing the on/off ratios, as charge transport is dominated by variable-range hopping. Experimentally, P3HT is often de-doped by soaking in ammonia-ethanol solutions [4], but this has proven insufficient for removing necessary amounts of oxygen. Here, we establish that use of long-duration low-temperature anneals is effective in oxygen removal. This was verified by elemental analysis via Rutherford Backscattering Spectrometry as shown in Table I and figure 3.

Table I: Sulfur and Oxygen concentrations as determined by RBS. Sulfur was used as a marker for the polythiophene layer.

\begin{tabular}{|c|c|}
\hline Sample & Composition \\
\hline control & 7.7at $\% \mathrm{~S} ; 15 \mathrm{at} \% \mathrm{O}$ \\
\hline $40^{\circ} \mathrm{C}$ & $7.5 \mathrm{at} \% \mathrm{~S} ; 12 \mathrm{at} \% \mathrm{O}$ \\
\hline $60^{\circ} \mathrm{C}$ & $\begin{array}{l}7.5 \text { at } \% \mathrm{~S} ; 12 \text { at } \% \text { O (O-rich } \\
\text { on the top } 2 \times 10^{17} / \mathrm{cm}^{2} \text { layer) }\end{array}$ \\
\hline $80^{\circ} \mathrm{C}$ & 7.0 at $\% \mathrm{~S} ; 6$ at $\% \mathrm{O}$ \\
\hline $100^{\circ} \mathrm{C}$ & $\begin{array}{l}7.0 \text { at } \% \mathrm{~S} ; 5 \text { at } \% \mathrm{O} \text { (slightly } \\
\text { more O-rich on the surface) }\end{array}$ \\
\hline $120^{\circ} \mathrm{C}$ & 6.5 at $\% \mathrm{~S}$ \\
\hline $140^{\circ} \mathrm{C}$ & 7.2 at $\% \mathrm{~S}$ \\
\hline
\end{tabular}

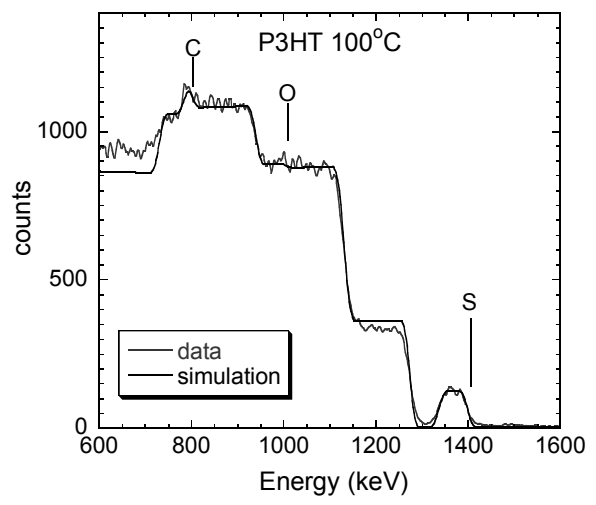

Figure 3: RBS Spectra for a typical sample, showing excellent fitting between the data and the parameter extraction signal.

Above $100^{\circ} \mathrm{C}$, oxygen concentrations were no longer detectable. The above data corresponds well to the on/off ratio: annealing at $40-60^{\circ} \mathrm{C}$ has little effect on oxygen concentration or on the on/off ratio, intermediate values of oxygen concentration ( $6 \mathrm{at} \%)$ correspond to intermediate levels of improvement in on/off ratio, and on/off ratios are maximized when oxygen concentration reaches its lowest levels. Note that the atomic concentrations were calibrated by fitting the RBS-spectra to the film thickness of unannealed films as measured by profilometry (which has an error of $\sim 15 \%$; this explains the sulfur concentration being slightly lower than the theoretically expected value sans 
hydrogen, which is not detected by RBS). The reduction in sulfur concentration with increasing temperature is related to a decrease in film density (discussed below), which introduces an error in the calibration since it is based on the thickness of unannealed films. The substantial decrease in oxygen content relative to sulfur content, however, clearly attests to the oxygen dedoping. To further analyze this effect, it is illustrative to examine the correlation between saturation mobility and conductivity, shown in figure 4 .

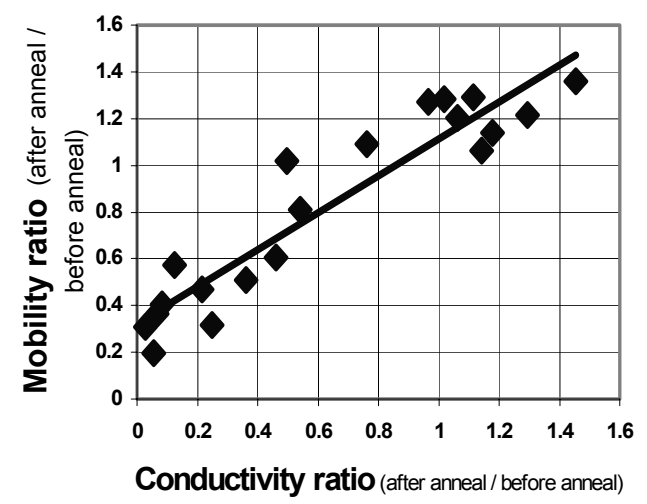

Figure 4: Correlation between saturation mobility and conductivity for all temperatures

The linear nature of the mobility vs. conductivity plot reaffirms that charge transport is controlled by variable-range hopping [3], and is therefore sensitive to oxygen dedoping. At higher mobilities, which correspond to lower annealing temperatures, the mobility to conductivity ratio is seen to decrease, which directly affects the on/off ratio:

$$
\text { Ion/Ioff }=(\mu / \sigma) \mathrm{C}_{\mathrm{i}}{ }^{2} \mathrm{~V}_{\mathrm{D}}{ }^{2} / \mathrm{qN}_{\mathrm{A}} \mathrm{t}^{2} \text { [3] }
$$

This ratio change and the decrease in acceptor density with annealing play key roles in the maximization of the on/off ratio of the P3HT transistors. Thus, the simultaneous increase in on-off ratio and decrease in mobility evident at intermediate temperatures $\left(60^{\circ} \mathrm{C}-120^{\circ} \mathrm{C}\right)$ is well explained using the oxygen de-doping mechanism.

\section{Chain Torsion}

While oxygen de-doping explains the intermediate temperature results well, it fails to explain the decrease in on/off ratio as anneal temperatures are raised above $120^{\circ} \mathrm{C}$. We explain this through the mechanism of conformational changes in the $\mathrm{P} 3 \mathrm{HT}$ film at elevated temperatures. Although the melting temperature for $\mathrm{P} 3 \mathrm{HT}$ is $178^{\circ} \mathrm{C}$, raising the film to a moderately close temperature is expected to thermodynamically allow a conformation change triggered by a conformation change within the alkyl-group. As deposited onto the substrate, $\mathrm{P} 3 \mathrm{HT}$ chains tend to lie flat. At elevated temperatures, the protruding alkyl chains cause torsioning along the backbone of the molecule. Due to the rigidity of the double bond, the most probable location for this twisting is at the bridging bonds between the thiophene rings $[5,6]$. In general, polythiophenes have relatively low rotational barriers, making the chains fairly flexible and increasing the likelihood of twisting along the backbone [7]. The torsioning of the P3HT chain decreases the conjugation length, therefore increasing the bandgap and drastically reducing the conductivity of the film. This physical change is irreversible, permanently harming the electrical characteristics of the device. Physical proof that the film is modified at raised 
temperatures is provided by AFM analysis, as shown in figure 5. RMS roughness measurements show an increase after higher annealing temperatures indicative of the rearrangement of the film into a more disorganized state.

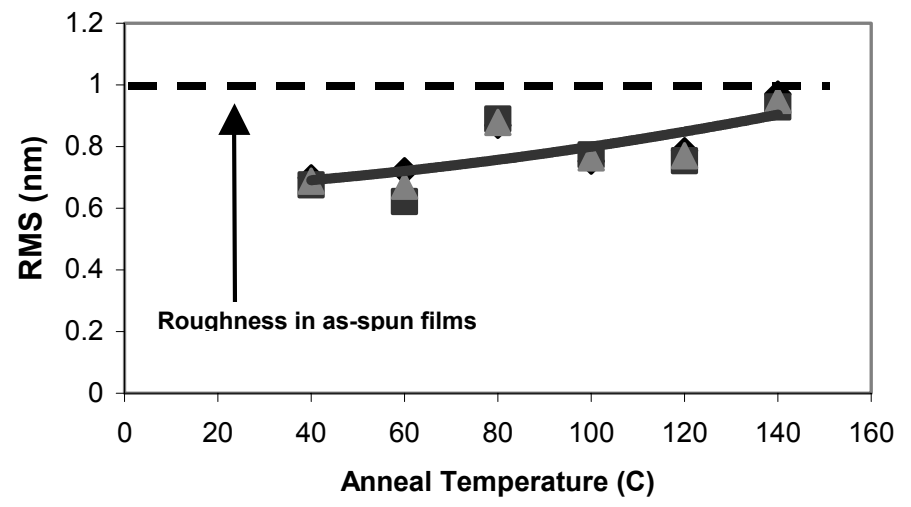

Figure 5: RMS roughness in P3HT films as a function of anneal temperature. The roughness in as-spun films is approximately $1 \mathrm{~nm}$ RMS.

\section{Drying and densification}

The final result that cannot be described is the drastic increase in mobility (and oncurrent) after short-duration low temperature anneals. No change in oxygen concentration occurs, yet for even a one-minute anneal at $60^{\circ} \mathrm{C}$ the mobility increases by $\sim 40 \%$. For longer anneals the effect is more pronounced, and doubles the pre-anneal mobility. The probable reasoning for this is the driving off of moisture and remnant solvent molecules, and the densification of the as-spun film. Following the spin procedure, the film hardens due to the evaporation of the choloroform solvent. Substantial choloroform and some moisture may be trapped within the polythiophene film. It is possible that low-temperature thermal annealing assists in the evolution of this, increasing performance; however, the low-temperatures involved make it unlikely that this is the sole basis for performance enhancement, since evolution at this temperature is likely to be minimal. An alternative explanation is the densification / reordering of the polythiophene material itself. As spun, film ordering is poor. Lowtemperature annealing may improve the ordering of the material, enhancing mobility. Some hint of this is provided by the reduction in RMS roughness achieved at low temperatures over that in as-spun films. Currently, we are unable to isolate the specific mechanism involved in the initial improvement in device performance, and further experiments are underway to clarify the nature of the underlying physical phenomena.

\section{DISCUSSION}

From the experiments above, it is apparent that the performance of poly(3hexylthiophene) transistors can be altered dramatically as a consequence of exposure to elevated temperatures. In particular, thermal annealing may represent an attractive means of improving the initial quality of organic TFTs, analogous to the annealing processes typically used with inorganic semiconductors. Thermal annealing is highly effective in removing oxygen contamination from polythiophene active layers, resulting in a dramatic 
improvement in on-off ratio. Given the potential applications of such devices in low-cost displays and low-power RFID circuits, high on-off ratio is a critical requirement.

It is also possible to analyze the stability and reliability of polythiophene TFTs based on the results above. As described previously, elevated temperatures cause structural changes in the polythiophene films, leading to degradation in both mobility and on-off ratio. This change is irreversible, and is indicative of damage to the thiophene backbone structure. Such changes happen above $120^{\circ} \mathrm{C}$, which represents an upper limit on the thermal excursion allowed in polythiophene-based devices. This has consequences for various integration strategies - for example, the use of post-thiophene polyimide processes for dielectrics and passivation is not possible, since these typically require imidization temperatures above $150^{\circ} \mathrm{C}$. As an additional consequence of the thermal analyses above, further experiments should enable the calculation of activation energies associated with the thermal film disruption processes, which should allow the development of a metric for accelerated lifetime testing of these devices.

\section{CONCLUSIONS}

The electrical and physical effects of thermal cycling on Poly(3-hexylthiophene) transistors has been studied. It has been shown that device performance can be enhanced through low-temperature annealing. Observations of the thermally-induced variations in device parameters have also led to insights into the physical effects occurring inside the semiconducting film. Among them, the oxygen dedoping of P3HT films has been indicated as a dominant force in driving changes in device performance. Meanwhile, transformations in conjugation length that occur near the melting temperature establish the thermal limits of the material. This analysis provides a basis for integration of P3HT with other low-cost processes, and established a baseline set of phenomena determining the thermal reliability of the same.

\section{ACKNOWLEDGEMENTS}

Support for this work came from the MARCO Focused Research Center on Materials, Structures, and Devices, in part by MARCO under contract 2001-MT-887 and DARPA under grant MDA972-01-1-0035.

\section{REFERENCES}

[1] H. Sirringhaus, N. Tessler, and R. H. Friend, Science 280, 1741 (1998)

[2] C. Vaterlein, B. Ziegler, W. Gebauer, H. Neureiter, M. Stoldt, M.Weaver, Synthetic Metals 76, 133 (1996)

[3] A. Brown, C. Jarrett, D. Leeuw and M. Matters, Synthetic Metals 88, 37 (1997)

[4] Y. Liu, K. Oshima, T. Yamauchi, M. Shimomura and S. Miyauchi, Synthetic Metals 101, 451 (1999)

[5] K. Yoshino, D. Park, B. Park, M. Onoda and R. Sugimoto, Solid State Comm. 67, 1119 (1988)

[6] S. Hotta and K. Ito "Electronic Properties of Polythiophenes.”Denis Fichou, Handbook of Oligo- and Polythiophenes, ed. D. Fichou (Wiley-vch, 1999) pp. 45-82

[7] G. Kilic, L. Toppare and E. Yurtsever. Synthetic Metals 79, 19 (1996) 